\title{
Long-term follow-up until early adulthood in autosomal dominant, complex SPG30 with a novel KIF1A variant: a case report
}

\author{
Carlotta Spagnoli ${ }^{1 *}$ D, Susanna Rizzi ${ }^{1}$, Grazia Gabriella Salerno ${ }^{1}$, Daniele Frattini ${ }^{1}$ and Carlo Fusco ${ }^{1,2}$
}

\begin{abstract}
Background: Pathogenic variants in KIF1A (kinesin family member 1A) gene have been associated with hereditary spastic paraplegia (HSP) type 30 (SPG30), encopassing autosomal dominant and recessive, pure and complicated forms.

Case presentation: We report the long-term follow-up of a 19 years-old boy first evaluated at 18 months of age because of toe walking and unstable gait with frequent falls. He developed speech delay, mild intellectual disability, a slowly progressive pyramidal syndrome, microcephaly, bilateral optic subatrophy and a sensory axonal polyneuropathy. Brain MRI showed cerebellar atrophy, stable along serial evaluations (last performed at 18 years of age). Targeted NGS sequencing disclosed the de novo c.914C > T missense, likely pathogenic variant on KIF1A gene.

Conclusions: We report on a previously unpublished de novo heterozygous likely pathogenic KIF1A variant associated with slowly progressive complicated SPG30 and stable cerebellar atrophy on long-term follow-up, adding to current knowledge on this HSP subtype.
\end{abstract}

Keywords: Cerebellar atrophy, Hereditary spastic paraplegia, SPG30, KIF1A

\section{Background}

Hereditary spastic paraplegias (HSP) are a group of clinically and genetically heterogeneous neurodegenerative disorders featuring progressive loss of corticospinal motor tract function, resulting in spasticity predominantly in the lower limbs, which can be isolated (pure forms) or associated with additional neurological or non-neurological features (complex forms) $[1,2]$.

Pathogenic variants in KIF1A were first associated with two neurodegenerative diseases in 2011: hereditary sensory and autonomic neuropathy (HSAN) type 2 [3] and a form of AR hereditary spastic paraparesis (HSP) [4]. One year later, it was demonstrated that KIF1A pathogenic variants cause SPG30 [5], and in the following years, both AR [6] and AD $[7,8]$, pure and complicated [7, 9] forms of HSP were described in association with KIF1A pathogenic variants. A

\footnotetext{
* Correspondence: carlotta.spagnoli@gmail.com

${ }^{1}$ Neuropsichiatria Infantile, Presidio Ospedaliero Provinciale S. Maria Nuova,

Azienda USL-IRCCS di Reggio Emilia, Reggio Emilia, Italy

Full list of author information is available at the end of the article
}

third phenotype (mental retardation, autosomal dominant 9) (OMIM \#614255), initially thought to represent a distinct disease, has been later demonstrated to be also caused by KIF1A pathogenic variants.

Description of long-term follow-up data on patients with rare neurogenetic conditions can be crucial to increase our knowledge on the natural history of the disease and to correctly disentangle it from phenotypic heterogeneity [10].

We present the clinical, neuroimaging and genetic data on a patient recently diagnosed with SPG30 with longterm neurologic follow-up, ranging from infancy to early adulthood.

\section{Case presentation}

A male patient, now aged 19 years, was born at term after uneventful pregnancy and delivery (Apgar scores: 9, 9, birth weight $3680 \mathrm{~g}$ ), from healthy non-consanguineous Caucasian parents. He has an older sister reportedly toe walking in the first 2 years of life. His early developmental milestones were normally achieved (independent sitting at 6 
months, first words at 12 months). He made his first steps at 14 months, however, concerns arose regarding toe walking and unstable gait with frequent falls, and he was first referred to child neurology evaluation at 18 months of age. Pyramidal signs at his lower limbs were detected. Subsequently, he developed speech delay, learning difficulties, and mild intellectual disability. During follow-up, microcephaly, bilateral optic subatrophy, hyperlordosis and asymmetric equino-valgo-pronated feet also became evident. Moreover, his spastic paraparesis has shown a slowly progressive course, with increasing difficulties in independent walking and a worsening in his hypertonus in the lower limbs. No quantitative scale was administered during follow-up.

At the last follow-up, he is slightly microcephalic $(\mathrm{OFC}=53 \mathrm{~cm},<-2 \mathrm{SD})$. He shows toe walking with a broad-based gait and valgo-pronated feet.

During this long follow-up, he underwent extended diagnostic investigations. Brain MRI, first performed at 18 months of age, documented superior vermis atrophy, and reduced volume of the optic nerves and optic chiasm, with no significant progression (last examined at 18 years of age) (Fig. 1).

Somatosensory evoked potentials at 12 years of age disclosed asymmetrically increased latencies of the central sensory conduction velocity. Serial ENG/EMG tests initially showed normal results, but at 14 years of age a sensory axonal polyneuropathy was diagnosed, showing evolution towards sensory-motor involvement at all four limbs (aged 18 years).

Negative neurometabolic and neuromuscular investigations include alpha-fetoprotein, amino acids, beta-exosaminidase, muscle biopsy with immuno-hystochemistry, and respiratory chain enzimes activity. Neurogenetically-wise, direct sequencing of POLG, Twinkle, PLA2G6, OPA1, OPA3, SPG20, and SCA5 retrieved negative results, while two known polymorphisms were detected on the exons 11 and 15 of the SPG7 gene. Array-CGH detected two partial duplications, both of maternal origin: arr3q29(197,728,559-197,840,339) x3mat (including the $L M L N$ gene) and arr4q22.1(93,202,223-93,359, 983) x3mat (including the GRID2 gene).

A targeted NGS panel for hereditary spastic paraplegia and motoneuronal disorders disclosed the heterozygous candidate variant 241,715,312 NM_001244008.1 c.914C > T (NP_001230937.1 p.Pro305Leu) on KIF1A gene, arisen de novo.

\section{Discussion and conclusions}

The variant we identified is a previously unpublished in the literature, missense, non-tolerated variant, absent in controls. It has been previously reported in ClinVar in four cases, in two in association with mental retardation, autosomal dominant 9 (OMIM \#614255), as a de novo variant, interepreted as likely pathogenic in the first patient and as pathogenic in the second one. Of note, the third and fourth cases report it as pathogenic, and in one the associated phenotype is characterized by developmental delay, cerebellar atrophy, ataxia, and eye movement abnormalities (patient tested at GeneDx). The variant is located inside the motor domain of kine$\sin 1 \mathrm{~A}$, in the $\mathrm{N}$ terminus of the protein (aa 1-365), where the vast majority of pathogenic variants associated with both AR and AD SPG30 are clustered. This domain

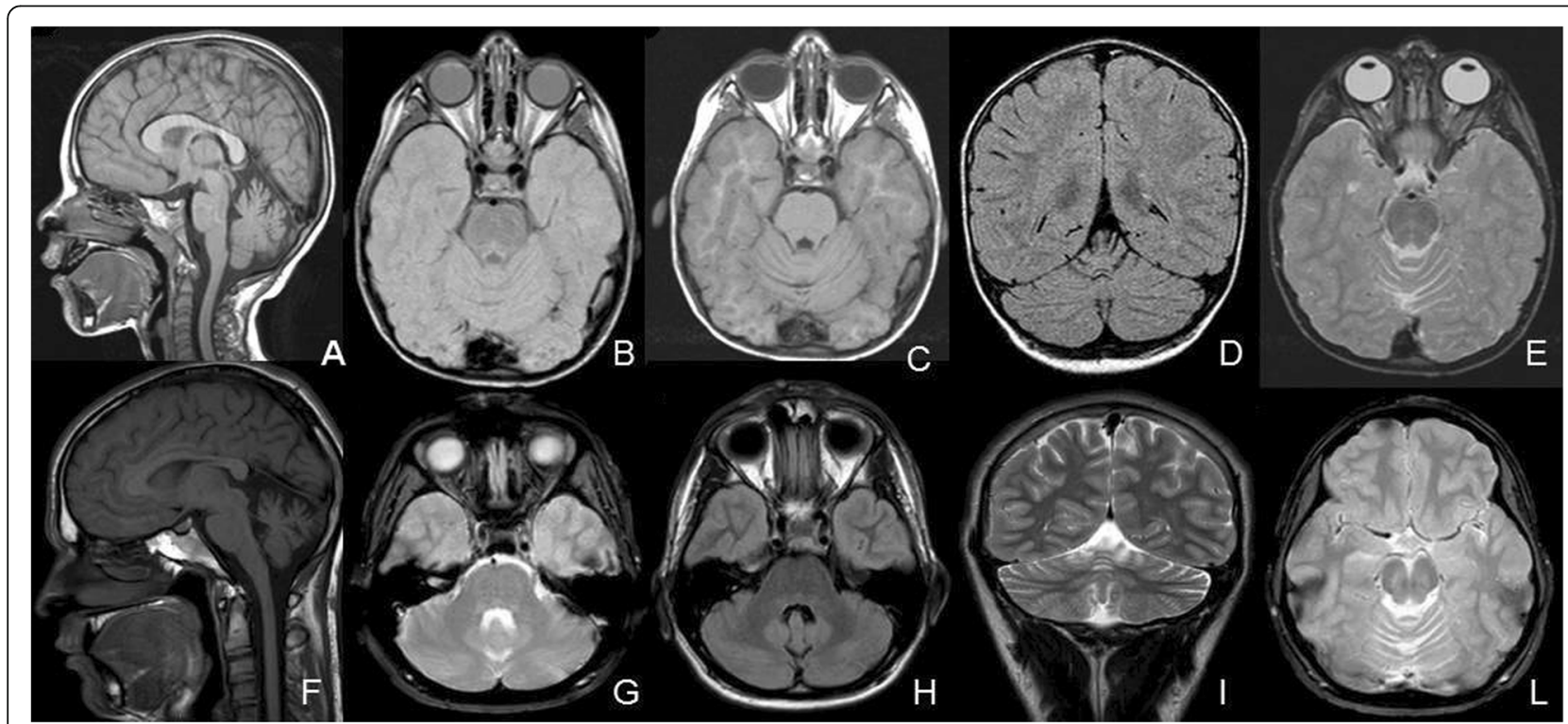

Fig. 1 Brain MRI at 4 (first row) and18 (second row) years of age, showing stable superior vermis atrophy and reduced volume of the optic nerves. $\mathbf{a}$ and $\mathbf{f}$ : sagittal T1 sequences, $\mathbf{b}$ and $\mathbf{g}$ : axial T2 sequences, $\mathbf{c}$ and $\mathbf{h}$ : longitudinal FLAIR sequences, $\mathbf{d}$ : coronal FLAIR and $\mathbf{i}$ : coronal T2 sequences, e and I: longitudinal T2 sequences 
is necessary for kinesin 1A movement along neurites and for binding with ATP and microtubules [11]. The p.Pro305Leu variant is a semi-conservative amino acid substitution, which may impact on secondary protein structure. This substitution occurs at a position which is conserved across species, and in silico analysis predicts it as probably damaging.

The variant is classified as likely pathogenic according to ACMG criteria [12]: PM1: Located in a mutational hot spot and/or critical and well-established functional domain (e.g., active site of an enzyme) without benign variation; PM2: Absent from controls (or at extremely low frequency if recessive) in Exome Sequencing Project, 1000 Genomes Project, or Exome Aggregation Consortium; PP3: Multiple lines of computational evidence support a deleterious effect on the gene or gene product (conservation, evolutionary, splicing impact, etc.); PP5: Reputable source recently reports variant as pathogenic, but the evidence is not available to the laboratory to perform an independent evaluation (InterVar, last examined 10th October 2019).

The clinical picture associated with complicated forms of SPG30 typically features intellectual disability, along with cerebellar atrophy and spastic paraplegia, with very wide range of onset. In some cases, optic nerve atrophy, thinning of the corpus callosum, periventricular white matter lesions, epilepsy and microcephaly (in two literature patients as well as the present one) [9], have also been described. The broad spectrum of observed clinical features likely reflects the ubiquitous expression of KIF1A in the nervous system, with a key role in anterograde transport of synaptic vescicles along axons [13]. Here we report a case of complicated, AD-SPG30 associated with a novel candidate missense variant located in the motor domain and characterized by the typical clinical phenotype associating spasticity, intellectual disability, cerebellar involvement and neuropathy [6].

Clinically, in contrast with the first descriptions of heterozygous cases, initially reported to be associated to both younger age at onset and more severe phenotypes [14], our patient has shown a slow progression of his pyramidal syndrome in his first 19 years of life, while cerebellar atrophy has remained unchanged up to 18 years (Fig. 1). Cerebellar atrophy is an almost constant finding in complicated forms of SPG30, but the majority of studies lack data on its temporal evolution. Hotchkiss and colleagues described two unrelated patients with progressive cerebellar atrophy demonstrated on sequential brain MRI's performed between 11 months and 12.5 years in the first and between 6 months and 6 years in the second one. Interestingly, these patients had normal cerebellar size when first imaged in infancy [15]. To the best of our knowledge, the oldest published patient with serial brain MRI's is a 27 years old female patient with no progression of cerebellar atrophy between 11 and 26 years of age [14]. In our case, there is no progression in cerebellar atrophy in the long-term follow-up from infancy to early adulthood, even though the patient harbours a variant in the heterozygous state.

In summary, with long-term follow-up, this case adds to current knowledge on phenotypic and genotypic variability in complex AD SPG30.

\begin{abstract}
Abbreviations
AD: Autosomal dominant; AR: Autosomal recessive; Array-CGH: arraycomparative genomic hybridization; ATP: Adenyl-triphosphate;

EMG: Electromyography; ENG: Electroneurography; g: grams;

GRID2: Glutamate receptor ionotropic delta 2; HSAN: Hereditary sensory and autonomic neuropathy; HSP: Hereditary spastic paraplegia; KIF1A: Kinesin family member 1A; LMLN: Leishmanolysin-Like (Metallopeptidase M8 Family); MRI: Magnetic resonance imaging; NGS: Next generation sequencing; NM: mRNA in Reference Sequence; NP: Protein in Reference Sequence; OFC: Occipito-frontal circumference; OMIM: Online Mendelian Inheritance in Man; OPA1: Optic atrophy 1; OPA3: Optic atrophy 3; PLA2G6: Phospholipase A2 group VI; POLG: Polymerase gamma; SCA5: Spinocerebellar ataxia type 5; SD: Standard deviation; SPG20: Spastic paraplegia type 20; SPG30: Spastic paraplegia type 30; SPG7: Spastic paraplegia type 7
\end{abstract}

\section{Acknowledgements}

No acknowledgements.

\section{Authors' contributions}

CS wrote the manuscript draft, collected the clinical data and approved the manuscript in its final form, SR, GGS and DF critically reviewed the manuscript and approved it in its final form, CF conceived the study design, critically reviewed the draft for intellectual content, and approved the manuscript in its final form.

\section{Funding}

No funding was secured for the completion of this study.

Availability of data and materials

Data and materials will be made available upon request.

Ethics approval and consent to participate

No ethics committee approval was required.

Consent for publication

Consent for publication was given.

\section{Competing interests}

The authors declare that they have no competing interests.

\section{Author details}

${ }^{1}$ Neuropsichiatria Infantile, Presidio Ospedaliero Provinciale S. Maria Nuova, Azienda USL-IRCCS di Reggio Emilia, Reggio Emilia, Italy. ${ }^{2}$ SC Neuropsichiatria Infantile Laboratorio di Neurofisiologia dell'Età Evolutiva. Presidio Ospedaliero Provinciale S. Maria Nuova, Azienda USL-IRCCS di Reggio Emilia, Reggio Emilia, Italy.

Received: 6 August 2019 Accepted: 21 November 2019

Published online: 03 December 2019

\section{References}

1. Fusco C, Frattini D, Farnetti E, et al. Hereditary spastic paraplegia and axonal motor neuropathy caused by a novel SPG3A de novo mutation. Brain and Development. 2010;32(7):592-4.

2. Fusco C, Frattini D, Farnetti $E$, et al. Very early onset and severe complicated phenotype caused by a new spastic paraplegia 3A gene mutation. J Child Neurol. 2012 Oct;27(10):1348-50.

3. Rivière $J B$, Ramalingam $S$, Lavastre $V$, et al. KIF1A, an axonal transporter of synaptic vesicles, is mutated in hereditary sensory and autonomic neuropathy type 2. Am J Hum Genet. 2011 Aug 12;89(2):219-30. 
4. Erlich $\mathrm{Y}$, Edvardson $\mathrm{S}$, Hodges $\mathrm{E}$, et al. Exome sequencing and diseasenetwork analysis of a single family implicate a mutation in KIF1A in hereditary spastic paraparesis. Genome Res. 2011;21(5):658-64.

5. Klebe S, Lossos A, Azzedine H, et al. KIF1A missense mutations in SPG30, an autosomal recessive spastic paraplegia: distinct phenotypes according to the nature of the mutations. Eur J Hum Genet. 2012;20(6):645-9.

6. Krenn M, Zulehner G, Hotzy C, et al. Hereditary spastic paraplegia caused by compound heterozygous mutations outside the motor domain of the KIF1A gene. Eur J Neurol. 2017;24(5):741-7.

7. Citterio A, Arnoldi A, Panzeri E, et al. Variants in KIF1A gene in dominant and sporadic forms of hereditary spastic paraparesis. J Neurol. 2015;262(12): 2684-90.

8. Roda RH, Schindler AB, Blackstone C. Multigeneration family with dominant SPG30 hereditary spastic paraplegia. Ann Clin Transl Neurol. 2017;4(11):821-4.

9. Cheon CK, Lim SH, Kim YM, et al. Autosomal dominant transmission of complicated hereditary spastic paraplegia due to a dominant negative mutation of KIF1A, SPG30 gene. Sci Rep. 2017;7(1):12527.

10. lodice A, Panteghini C, Spagnoli C, et al. Long-term follow-up in spastic paraplegia due to SPG56/CYP2U1: age-dependency rather than genetic variability? J Neurol. 2017;264(3):586-8.

11. Shin H, Wyszynski M, Huh KH, et al. Association of the kinesin motor KIF1A with the multimodular protein liprin-alpha. J Biol Chem. 2003;278(13): 11393-401.

12. Richards S, Aziz N, Bale SJ, et al. Standards and guidelines for the interpretation of sequence variants: a joint consensus recommendation of the American College of Medical Genetics and Genomics and the Association for Molecular Pathology. Genetics in Medicine. 2015;17(5):405-24.

13. Hirokawa N, Nitta R, Okada Y. The mechanisms of kinesin motor motility: lessons from the monomeric motor KIF1A. Nat Rev Mol Cell Biol. 2009; 10(12):877-84.

14. Ohba C, Haginoya K, Osaka H, et al. De novo KIF1A mutations cause intellectual deficit, cerebellar atrophy, lower limb spasticity and visual disturbance. J Hum Genet. 2015:60(12):739-42.

15. Hotchkiss L, Donkervoort S, Leach ME, et al. Novel de novo mutations in KIF1A as a cause of hereditary spastic paraplegia with progressive central nervous system involvement. J Child Neurol. 2016;31(9):1114-9.

\section{Publisher's Note}

Springer Nature remains neutral with regard to jurisdictional claims in published maps and institutional affiliations.

Ready to submit your research? Choose BMC and benefit from:

- fast, convenient online submission

- thorough peer review by experienced researchers in your field

- rapid publication on acceptance

- support for research data, including large and complex data types

- gold Open Access which fosters wider collaboration and increased citations

- maximum visibility for your research: over $100 \mathrm{M}$ website views per year

At BMC, research is always in progress.

Learn more biomedcentral.com/submissions 